\title{
Entanglement-assisted quantum communication beating the quantum Singleton bound
}

\author{
Markus Grass1 (1) \\ International Centre for Theory of Quantum Technologies, University Gdansk, 80-308 Gdansk, Poland \\ and Max-Planck-Institut für die Physik des Lichts, 91058 Erlangen, Germany
}

(Received 2 July 2020; revised 3 February 2021; accepted 5 February 2021; published 24 February 2021)

\begin{abstract}
Brun, Devetak, and Hsieh [Science 314, 436 (2006)] demonstrated that preshared entanglement between the sender and receiver enables quantum communication protocols that have better parameters than schemes without the assistance of entanglement. Subsequently, the same authors derived a version of the so-called quantum Singleton bound that relates the parameters of the entanglement-assisted quantum-error-correcting codes proposed by them. We present an entanglement-assisted quantum communication scheme with parameters violating this bound in certain ranges. For a fixed transmission rate, our scheme allows one to correct a larger fraction of errors.
\end{abstract}

DOI: 10.1103/PhysRevA.103.L020601

Introduction. Entanglement is a resource that enables or enhances many tasks in quantum communication. When the sender and receiver share a maximally entangled state, quantum teleportation allows the sender to transmit an unknown quantum state by just sending a finite amount of classical information over a noiseless classical channel [1]. When the entangled states are initially distributed over a noisy quantum channel, using local quantum operations and a noiseless classical channel, the sender and receiver can extract a smaller number of maximally entangled states with higher fidelity by a distillation process [2]. The correspondence between entanglement distillation protocols and quantum-error-correcting codes in a communication scenario has been discussed in Ref. [3]. Quantum-error-correcting codes (QECCs), however, are somewhat more general in the sense that they allow one to recover a quantum state affected by a general quantum channel, provided that a suitable encoding for that channel exists [4]. Hence QECCs can be used both for communication and storage, and they are essential ingredients for fault-tolerant quantum computation [5]. On the other hand, in Ref. [6] it has been shown that the performance of QECCs in a communication scenario can be improved when a noisy quantum channel is assisted by entanglement.

In this Letter we present a quantum communication scheme that also uses a noisy quantum channel assisted by entanglement. The main idea is to execute a teleportation protocol in which the classical information is protected using a code and then sent via the noisy quantum channel to the receiver. This allows one to use classical error-correcting codes. In some range, our scheme has better parameters than

Published by the American Physical Society under the terms of the Creative Commons Attribution 4.0 International license. Further distribution of this work must maintain attribution to the author(s) and the published article's title, journal citation, and DOI. Open access publication funded by the Max Planck Society. the one proposed in Ref. [6], showing that the adaption of the quantum Singleton bound to that class of codes presented in Ref. [7] can be violated. The original bound was based on the implicit assumption that the so-called no-cloning bound [8] applies to entanglement-assisted quantum codes as well. The no-cloning bound states that for a quantum code of dimension $K>1$, the number of correctable erasures is strictly less than half the length of the code; otherwise one would be able to split the code into two subsystems and then perfectly recover the quantum information independently in both of the systems. Our scheme demonstrates that with the assistance of preshared entanglement, one can correct more errors. This is no contradiction to the impossibility of perfect cloning [9], as the preshared entanglement can only be used once in the error-recovery procedure.

Quantum teleportation. We start with a short summary of quantum teleportation [1], as illustrated in Fig. 1.

The aim is to transmit an arbitrary quantum state $|\varphi\rangle$ in the Hilbert space $\mathscr{H} \cong\left(\mathbb{C}^{q}\right)^{\otimes c}$ of $c$ quantum systems of dimension $q$ (qudits [10]). The protocol is assisted by $c$ copies of a maximally entangled bipartite state

$$
|\Phi\rangle_{S R}=\frac{1}{\sqrt{q}} \sum_{i=0}^{q-1}|i\rangle_{S}|i\rangle_{R},
$$

which are shared by sender $S$ and receiver $R$. Applying Heisenberg-Weyl operators $X^{a} Z^{b}$ (or generalized Pauli matrices) on one of the systems, the collection of the resulting states constitutes the generalized Bell basis

$$
\left\{\left|\Phi^{a, b}\right\rangle=\left(I \otimes X^{a} Z^{b}\right)|\Phi\rangle: a, b=0, \ldots, q-1\right\} .
$$

Here, $X$ is a cyclic shift operator in the standard basis and $Z$ its diagonal form.

The sender performs a generalized Bell measurement, i.e., a measurement in the generalized Bell basis (2), on the input state $|\varphi\rangle$ and $c$ qudits from the maximally entangled states. For each pair of qudits, one obtains a pair $\left(a_{i}, b_{i}\right)$ of classical values. The strings $\mathbf{a}=\left(a_{1}, a_{2}, \ldots, a_{c}\right)$ and $\mathbf{b}=\left(b_{1}, b_{2}, \ldots, b_{c}\right)$ 


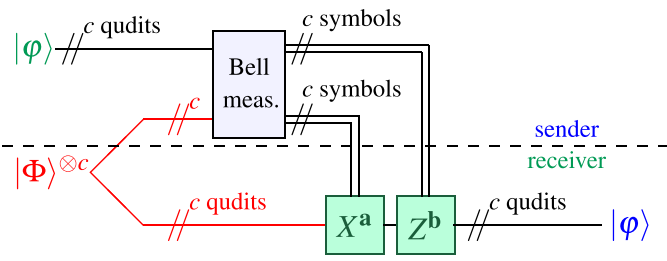

FIG. 1. Teleportation protocol. The maximally entangled states $|\Phi\rangle^{\otimes c}$ can be prepared by either party, or even a third party.

with $c$ classical symbols each are sent to the receiver, indicated by the double lines in Fig. 1. Depending on the values of a and $\mathbf{b}$, the receiver applies correction operations $X^{\mathbf{a}}=X^{a_{1}}$ $\otimes \cdots \otimes X^{a_{c}}$ and $Z^{\mathbf{b}}=Z^{b_{1}} \otimes \cdots \otimes Z^{b_{c}}$ and obtains the original state $|\varphi\rangle$.

Quantum-error-correcting codes. A standard quantumerror-correcting code $\mathscr{C}$ of length $n$ is a subspace of the Hilbert space $\left(\mathbb{C}^{q}\right)^{\otimes n}$ of $n$ qudits. Usually, the local dimension $q$ is assumed to be a power of a prime, i.e., $d=p^{m}$ for some prime $p$ [11]. A QECC encoding $k$ qudits has dimension $q^{k}$ and is denoted by $\llbracket n, k, d \rrbracket_{q}$. A QECC with minimum distance $d=2 t+1$ allows one to correct all errors affecting no more than $t$ of the subsystems. When the position of the errors is known, then errors on up to $d-1$ subsystems can be corrected [12]. Alternatively, all errors on no more than $d-1$ of the subsystems that act nontrivially on the code can be detected. Independent of the dimension $q$ of the subsystems, the parameters of a QECC are constrained by the so-called quantum Singleton bound [4,13]

$$
2 d \leqslant n-k+2 \text {. }
$$

Codes meeting this bound with equality are called quantum $M D S$ (QMDS) codes, where the acronym MDS stands for maximum distance separable. With few exceptions, QMDS codes are only known for length $n \leqslant q^{2}+1$ and minimum distance $d \leqslant q+1$ [14].

The overall scheme is illustrated in Fig. 2. The unitary encoding operator $\mathscr{E}$ maps the input state $|\varphi\rangle$ with $k$ qudits and $n-k$ ancilla qudits $\left|0^{n-k}\right\rangle$ to the encoding space with $n$ qudits. Those $n$ qudits are sent over a noisy quantum channel $\mathscr{N}$, whose output enters the decoder $\mathscr{D}$. The decoder outputs a quantum state $\left|\varphi^{\prime}\right\rangle$ with $k$ qudits. When the error can be corrected, the states $\left|\varphi^{\prime}\right\rangle$ and $|\varphi\rangle$ are equal.

Entanglement-assisted quantum-error-correcting codes. An entanglement-assisted quantum-error-correcting code (EAQECC), denoted by $\llbracket n, k, d ; c \rrbracket_{q}$, is a quantum-errorcorrecting code that additionally uses $c$ maximally entangled states. The scheme is illustrated in Fig. 3.

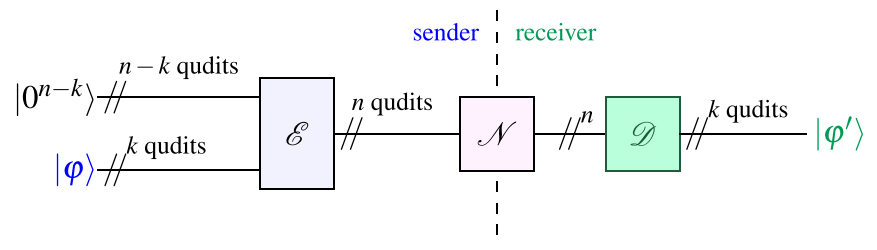

FIG. 2. Scheme of a communication protocol using a quantumerror-correcting code $\llbracket n, k, d \rrbracket_{q}$.

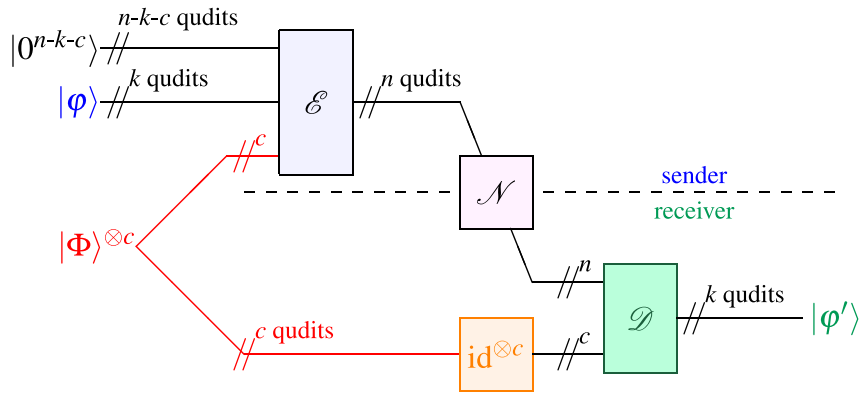

FIG. 3. Communication scheme using an entanglement-assisted quantum code $\llbracket n, k, d ; c \rrbracket_{q}$.

One half of each maximally entangled state $|\Phi\rangle \in \mathbb{C}^{q} \otimes$ $\mathbb{C}^{q}$ enters the encoding operation $\mathcal{E}$ together with the $k$-qudit state $|\varphi\rangle$ to be transmitted and $n-k-c$ ancilla qudits. The other half of each maximally entangled state is assumed to be transmitted error-free. The $n$ qudits output by the encoding operation are sent through the noisy quantum channel $\mathscr{N}$. The receiver applies the decoding operation $\mathscr{D}$ to both the $n$ qudits output from the channel and the $c$ noiseless qudits from the maximally entangled states.

In Ref. [7], the authors formulate a Singleton bound for the parameters $\llbracket n, k, d ; c \rrbracket_{q}$ of an EAQECC:

$$
2 d \leqslant n-k+2+c .
$$

This bound can be derived by considering the $n$ qudits sent over the noisy quantum channel $\mathcal{N}$ together with the $c$ qubits from the maximally entangled states sent over a noiseless channel as a standard QECC of length $n+c$ in the quantum Singleton bound (3). This, however, ignores the fact that the $c$ additional qudits are assumed to be error-free. The approach in Ref. [15] accounts for this additional assumption. In Theorem 6 of Ref. [15], the bound (4) has been shown to be valid for the case $d \leqslant(n+2) / 2$, which is the no-cloning bound mentioned in the Introduction.

In Ref. [16], EAQECCs meeting the bound (4) with equality were constructed. Some of the codes use only $c=1$ or $c=2$ maximally entangled states, and the maximal value is $c=q+1$. While the length of the codes is bounded by $q^{2}+1$ as in the case of QMDS codes, the minimum distance can be as large as $d=2 q$ for some of the codes, compared to $d \leqslant q+1$ for most QMDS codes without entanglement assistance.

In Ref. [6], a construction of EAQECC from any linear code $[n, \boldsymbol{\kappa}, d]_{q^{2}}$ over the finite field $\mathbb{F}_{q^{2}}$ with $q^{2}$ was given. The parameters of the resulting EAQECC are $\llbracket n, 2 \kappa-n+$ $c, d ; c \rrbracket_{q}$, where the number $c$ of maximally entangled states depends on the classical code and is at most $n-\boldsymbol{\kappa}$. Using a result from Ref. [17] it follows that for $q \geqslant 3$, any classical linear code $[n, \kappa, d]_{q^{2}}$ can be converted into an EAQECC that requires the maximal amount of entanglement $c=n-\kappa$. The EAQECC has parameters $\llbracket n, \kappa, d ; n-\kappa \rrbracket_{q}$. From a classical MDS code $[n, \boldsymbol{\kappa}, n-\boldsymbol{\kappa}+1]_{q^{2}}$, we obtain an EAQECC with parameters $\llbracket n, k, n-k+1 ; n-k \rrbracket_{q}$, meeting the bound (4) with equality. Assuming the maximal value for $c=n-k$, the minimum distance of an EAQECC from this construction 


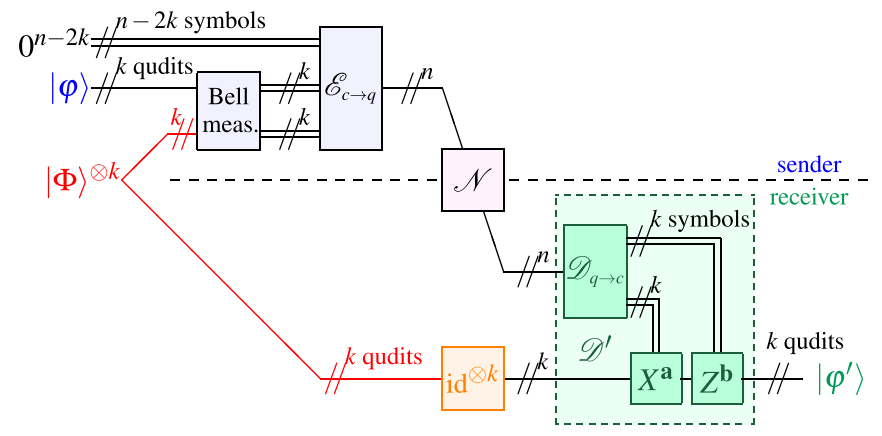

FIG. 4. Our teleportation-based scheme using $c=k$ maximally entangled states.

obeys the bound

$$
d \leqslant n-k+1,
$$

which is exactly the Singleton bound for classical codes.

The bound (5) is also an absolute bound on the minimum distance of any quantum code for the following reason. A quantum code with minimum distance $d$ can correct errors that affect $d-1$ errors at known positions. Hence, after tracing out $d-1$ of the systems, we will still be able to recover any encoded state of $k$ qudits. The residual state has $n-d+1$ qudits, and hence the bound $k \leqslant n-d+1$ follows.

The presented scheme. In our scheme, we use the $c$ maximally entangled states in a teleportation protocol to transmit $k=c$ qudits. Each generalized Bell measurement in the teleportation protocol has $q^{2}$ possible outcomes, i.e., we have to send a classical string with $2 k$ symbols from an alphabet of size $q$ to the receiver. As we allow for $n$ uses of a quantum channel, we can use a classical code $C$ over an alphabet of size $q$ encoding $2 k$ symbols into $n$ symbols, denoted by $[n, 2 k, d]_{q}$, where again $d$ denotes the minimum distance of the code (for more details about classical error-correcting codes, see, for example, Ref. [18]). The classical string of length $n$ is mapped to one of the $q^{n}$ basis states of the Hilbert space of $n$ qudits and then sent via the noisy quantum channel $\mathcal{N}$ to the receiver. The receiver measures the output of the quantum channel in the computational basis and obtains a classical string of length $n$. Applying error correction for the classical code $C$, the $2 k$ symbols corresponding to the measurement result from the teleportation protocol are retrieved. The measurement and the classical decoder are depicted together as a quantum-to-classical map $\mathscr{D}_{q \rightarrow c}$ in Fig. 4. The receiver applies the corresponding correction operators $X^{\mathbf{a}}$ and $Z^{\mathbf{b}}$ to the $c$ qudits from the $c$ maximally entangled states and completes the teleportation protocol.

The decoding operator $\mathscr{D}$ and the correction operators can be combined as a quantum map $\mathscr{D}^{\prime}$ (see the dashed box in Fig. 4). Furthermore, the sender does not have to perform a measurement in the generalized Bell basis, but may apply a unitary transformation that maps the Bell basis to the standard basis, labeled as "Bell transf." in Fig. 5. Then those $2 k$ qudits can be encoded into $n$ qudits using the quantum map $\mathscr{E}$. Hence, Fig. 5 shows a fully quantum version of our scheme, showing that our protocol uses the same type of operations as an EAQECC shown in Fig. 3. We will also use the same notation $\llbracket n, k, d ; c \rrbracket_{q}$ for the parameters.

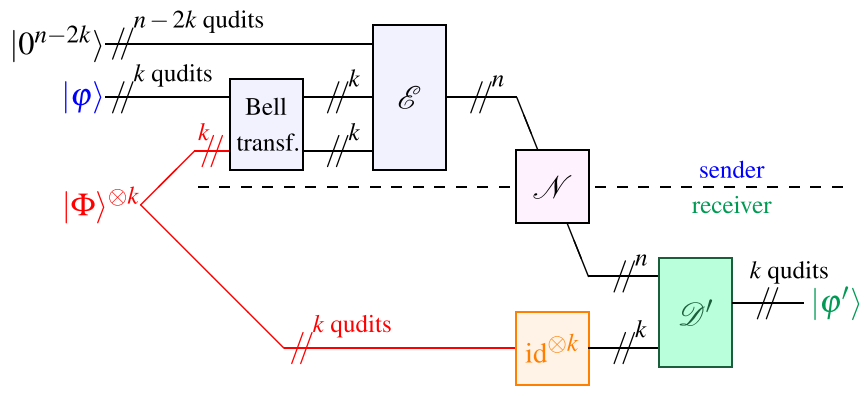

FIG. 5. Fully quantum version of the scheme shown in Fig. 4.

Using a linear classical code in our scheme, the resulting EAQECC can be described within the stabilizer formalism [19]. Similarly, the construction from Ref. [6] based on classical linear codes yields EAQECC within the stabilizer framework. On first glance, our scheme seems to be somewhat closer to Calderbank-Shor-Steane (CSS) codes [20,21]. The parity checks of the classical code correspond to $Z$-type stabilizers, and the additional stabilizer generators can be chosen to have mainly $X$-type operators.

On the other hand, note that we are only transmitting basis states over the quantum channel, and therefore the protocol is resilient to arbitrary phase errors. When following the standard teleportation protocol, as shown in Fig. 4, one can replace the quantum channel by a classical channel.

The parameters of our scheme are determined by the classical code $C$. The Singleton bound for classical codes implies the bound

$$
d \leqslant n-2 k+1
$$

on the minimum distance of our scheme. It can be achieved whenever the classical code is an MDS code. In the special case $k=c$, the bound (4) implies

$$
d \leqslant n / 2+1 \text {. }
$$

Hence, for $k<n / 4$ the bound (4) is more restrictive than the bound for our scheme (see also Fig. 6). Even when more maximally entangled states are used in the original construction of EAQECCs, our scheme has a larger normalized minimum distance $\delta=d / n$ for a rate $R=k / n$ below a certain threshold [e.g., $R<1 / 5$ for $c=(n-k) / 2$, see Fig. 6].

Examples. Classical MDS codes with parameters $[n, 2 k, n-2 k+1]_{q}$ are known to exist for $2 k \leqslant n \leqslant q+1$. Using such a code in our scheme results in parameters $\llbracket n, k, n-2 k+1 ; k \rrbracket_{q}$, meeting the bound (6) with equality. For prime powers $q \geqslant 4$, we have in particular classical MDS codes with parameters $[5,2,4]_{q}$, yielding a scheme $\llbracket 5,1,4 ; 1 \rrbracket_{q}$ using one maximally entangled state. In comparison, according to (4), the minimum distance $d$ would be at most 3. Standard QECCs with parameters $\llbracket 5,1,3 \rrbracket_{q}$ exist for all $q \geqslant 2$ and do not require preshared entanglement [22].

For the case $k=c=1$, i.e., transmitting one qudit with the help of a single maximally entangled state, we need a classical code with parameters $[n, 2, d]_{q}$. For $n \leqslant q+1$, an MDS code $[n, 2, n-1]_{q}$ exists. Repeating the code $[q+1,2, q]_{q} \ell$ times results in a classical code $[\ell(q+1), 2, \ell q]_{q}$ which is optimal by the Griesmer bound $[23,24]$. The parameters of the 


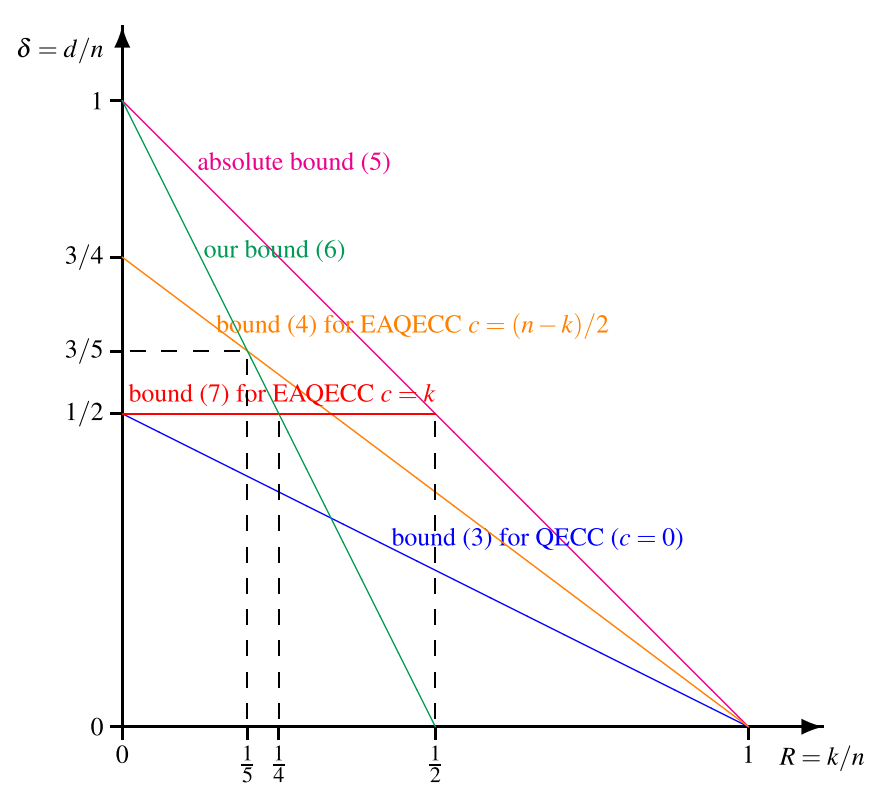

FIG. 6. Asymptotic bounds (length $n \rightarrow \infty$ ) on the normalized minimum distance $\delta=d / n$ as a function of the code rate $R=k / n$.

resulting entanglement-assisted communication scheme are $\llbracket \ell(q+1), 1, \ell q ; 1 \rrbracket_{q}$, again beating the bound (4). In particular, we get a scheme with parameters $\llbracket 9,1,6 ; 1 \rrbracket_{2}$ encoding a single qubit into nine qubits with the help of one EinsteinPodolsky-Rosen (EPR) pair [19]. The normalized minimum distance $\delta=q /(q+1)$ tends to 1 , while for a fixed amount $c$ of entanglement, the normalized minimum distance $\delta=d / n$ is bounded by $1 / 2$ in (4).

Concluding remarks. Quantum codes based on teleportation have been considered before when studying the entanglement-assisted capacity of quantum channels (see Sec. III E in Ref. [25]). It was observed that this results in an entanglement-assisted capacity that is half the classical capacity of the unassisted quantum channel. We are, however, not aware of related results for the finite-length case.

Our scheme beats the quantum Singleton bound (4) for quantum communication schemes with a rate below a certain threshold and uses a smaller amount $c$ of entanglement than the scheme proposed in Ref. [6]. On the other hand, when the amount of additional entanglement does not matter, using $c=n-k$ maximally entangled states in the original scheme reaches the absolute bound (5). In Fig. 3(c) of Ref. [26], it has been shown that for a fixed relative minimum distance $\delta=d / n>1 / 2$, using $c$ maximally entangled pairs with $k \leqslant$ $c \leqslant n-k$ results in a higher rate that grows linearly with $c$. But while our schemes uses $c=k$ maximally entangled pairs, that range requires even more entanglement. It is plausible to assume that using $c>n-k$ maximally entangled states would not result in better parameters, as in this case the encoding operation $\mathcal{E}$ would map $k+c>n$ qudits to a smaller number of qubits.

We conclude by noting that in order to beat the originally stated quantum Singleton bound for entanglement-assisted quantum-error-correcting codes (4), one has to use $c \geqslant$ $k$ maximally entangled pairs. This result, together with further bounds relating length $n$, dimension $k$, minimum distance $d$, and the number $c$ of maximally entangled pairs in general entanglement-assisted schemes can be found in Ref. [26].

Acknowledgments. The author acknowledges fruitful discussions with Frederic Ezerman, Min-Hsiu Hsieh, Felix Huber, Ching-Yi Lai, Hui Khoon Ng, Andreas Winter, and Bei Zeng. The "International Centre for Theory of Quantum Technologies" project (Contract No. 2018/MAB/5) is carried out within the International Research Agendas Programme of the Foundation for Polish Science cofinanced by the European Union from the funds of the Smart Growth Operational Programme, axis IV: Increasing the research potential (Measure $4.3)$.
[1] C. H. Bennett, G. Brassard, C. Crépeau, R. Jozsa, A. Peres, and W. K. Wootters, Teleporting an Unknown Quantum State via Dual Classical and Einstein-Podolsky-Rosen Channels, Phys. Rev. Lett. 70, 1895 (1993).

[2] C. H. Bennett, H. J. Bernstein, S. Popescu, and B. Schumacher, Concentrating partial entanglement by local operations, Phys. Rev. A 53, 2046 (1996).

[3] C. H. Bennett, D. P. DiVincenzo, J. A. Smolin, and W. K. Wootters, Mixed-state entanglement and quantum error correction, Phys. Rev. A 54, 3824 (1996).

[4] E. Knill and R. Laflamme, Theory of quantum error-correcting codes, Phys. Rev. A 55, 900 (1997).

[5] P. W. Shor, Fault-tolerant quantum computation, in Proceedings 37th Annual Symposium on Foundations of Computer Science (IEEE, New York, 1996), pp. 56-65.

[6] T. Brun, I. Devetak, and M.-H. Hsieh, Correcting quantum errors with entanglement, Science 314, 436 (2006).

[7] T. Brun, I. Devetak, and M.-H. Hsieh, Catalytic quantum error correction, IEEE Trans. Inf. Theory 60, 3073 (2014).
[8] N. J. Cerf and R. Cleve, Information-theoretic interpretation of quantum error-correcting codes, Phys. Rev. A 56, 1721 (1997).

[9] W. K. Wootters and W. H. Zurek, A single quantum cannot be cloned, Nature (London) 299, 802 (1982).

[10] The dimension is quite often denoted by the letter $d$, which in the context of coding theory is used to denote the minimum distance. To avoid ambiguity, we use the letter $q$ instead.

[11] When the local dimension $q$ is a power of a prime, the WeylHeisenberg operators are defined with respect to the finite field $G F(q)$ with $q$ elements instead of the integers modulo $q$ as before.

[12] M. Grassl, T. Beth, and T. Pellizzari, Codes for the quantum erasure channel, Phys. Rev. A 56, 33 (1997).

[13] E. M. Rains, Nonbinary quantum codes, IEEE Trans. Inf. Theory 45, 1827 (1999).

[14] M. Grassl and M. Rötteler, Quantum MDS codes over small fields, in Proceedings 2015 IEEE International Symposium on Information Theory (ISIT) (IEEE, New York, 2015), pp. 1104-1108. 
[15] C.-Y. Lai and A. Ashikhmin, Linear programming bounds for entanglement-assisted quantum error-correcting codes by split weight enumerators, IEEE Trans. Inf. Theory 64, 622 (2018).

[16] J. Fan, H. Chen, and J. Xu, Constructions of $q$-ary entanglement-assisted quantum MDS codes with minimum distance greater than $q+1$, Quantum Inf. Comput. 16, 423 (2016).

[17] C. Carlet, S. Mesnager, C. Tang, Y. Qi, and R. Pellikaan, Linear codes over $\mathbb{F}_{q}$ are equivalent to LCD codes for $q>3$, IEEE Trans. Inf. Theory 64, 3010 (2018).

[18] F. J. MacWilliams and N. J. A. Sloane, The Theory of ErrorCorrecting Codes (North-Holland, Amsterdam, 1977).

[19] M. Grassl, Entanglement-assisted quantum communication beating the quantum Singleton bound, presented at 16th Asian Quantum Information Science Conference (AQIS 2016), Taipei, Taiwan, 2016, available at http://aqis-conf.org/2016/program/.
[20] A. R. Calderbank and P. W. Shor, Good quantum errorcorrecting codes exist, Phys. Rev. A 54, 1098 (1996).

[21] A. M. Steane, Error Correcting Codes in Quantum Theory, Phys. Rev. Lett. 77, 793 (1996).

[22] H. F. Chau, Five quantum register error correction code for higher spin systems, Phys. Rev. A 56, R1 (1997).

[23] J. H. Griesmer, A bound for error-correcting codes, IBM J. Res. Dev. 4, 532 (1960).

[24] G. Solomon and J. J. Stiffler, Algebraically punctured cyclic codes, Inf. Control 8, 170 (1965).

[25] G. Bowen, Entanglement required in achieving entanglementassisted channel capacities, Phys. Rev. A 66, 052313 (2002).

[26] M. Grassl, F. Huber, and A. Winter, Entropic proofs of Singleton bounds for quantum error-correcting codes, arXiv:2010.07902. 